\title{
Insuficiência adrenal secundária associada a meningioma psamomatoso: relato de caso
}

\author{
Secondary adrenal insufficiency associated to psamomatoso meningioma: case report \\ Insuficiencia suprarrenal secundaria asociada a meningioma psamomatoso: reporte de \\ caso
}

Camila Maria Beder Ribeiro Girish Panjwani ${ }^{1 *}$, Dâmiris Camilo da Rocha ${ }^{1}$, Klysciane Ferreira da

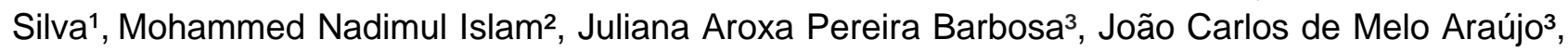
André Vasconcelos de Barros Lima ${ }^{4}$.

\section{RESUMO}

Objetivo: Avaliar um caso de Insuficiência Adrenal Secundária (IAS) associada a um Meningioma Psamomatoso (MP) na região da hipófise, analisando as manifestações orais da IAS, a relação entre essas patologias e discutir os dados clínicos com a literatura. Detalhamento do Caso: Participante da pesquisa, gênero feminino, 53 anos, compareceu ao Centro de Especialização Odontológica, com a queixa principal "Apareceu umas manchas escuras em minha boca". No exame intra-oral, foi observado uma lesão plana, rugosa, pigmentação melanocítica, localizada em mucosa jugal, língua e palato há 4 anos. $O$ histórico médico demonstra um meningioma do tipo psamomatoso na região da hipófise e passou por tratamento radioterápico e cirúrgico há 10 anos. Considerações finais: O IAS é uma patologia pouco frequente, não apresenta sintomas específicos, o que torna o diagnóstico difícil. O profissional da saúde deve realizar uma boa anamnese e ficar atento ao histórico do paciente.

Palavras-chave: Doenças da Boca, Insuficiência Adrenal, Hiperpigmentação, Neoplasias Encefálicas, Doenças da Hipófise.

\begin{abstract}
Objective: Evaluating a case of Secondary Adrenal Insufficiency (SAI) associated with a Psamomatous Meningioma (PM) in the pituitary region, analyzing the oral manifestations of SAI, the relationship between these pathologies and discuss the clinical data with the literature. Case Detailing: Female research participant, 53 years old, appeared on the Center for Dental Specialization, with the main complaint: "Dark spots appeared on my mouth." In the intra-oral examination, was observed a flat, rough and melanocytic pigmentation lesion, located in the jugal mucosa, tongue and palate for 4 years. The medical history demonstrates a psamomatous meningioma in the pituitary region and underwent radiotherapy and surgery 10 years ago. Final considerations: SAI is an uncommon pathology, with no specific symptoms, which makes the diagnosis difficult. The health professional should make a good history and be aware of the patient's history.
\end{abstract}

Key words: Mouth Diseases, Adrenal Insufficiency, Hyperpigmentation, Brain Neoplasms, Pituitary Diseases.

${ }^{1}$ Centro Universitário Cesmac, Maceió, Alagoas. *E-mail: cami.beder@gmail.com

2Universidade da Flórida, Flórida - Estados Unidos.

${ }^{3}$ Centro de Patologia de Maceió - CEPAMA, Maceió, Alagoas.

${ }^{4}$ Centro de Especialidades Odontológicas - CEO, Campo Alegre, Alagoas.

SUBMETIDO EM: 10/2019

ACEITO EM: 10/2019

PUBLICADO EM: 10/2019

REAS/EJCH | Vol.11(16) | e2014 | DOI: https://doi.org/10.25248/reas.e2014.2019 Página 1 de 6 


\section{RESUMEN}

Objetivo: Evaluar un caso de Insuficiencia Adrenal Secundaria (IAS) asociada a un Meningioma Psamomatoso (MP) en la región de la hipófisis, analizando las manifestaciones orales de la IAS, la relación entre esas patologías y discutir los datos clínicos con la literatura. Detalles del caso: Participante del caso, género femenino, 53 años, compareció al Centro de Especialización Odontológica, con la queja principal: "aparecieron unas manchas oscuras en mi boca". En el examen intra-oral, fue observado una lesión plana, rugosa, pigmentación melanocítica, localizada en la mucosa jugal, lengua y palato hace 4 años. La historia clínica demuestra un meningioma del tipo psamomatoso en la región de la hipófisis y, que pasó por tratamiento radioterápico y cirúrgico, hace 10 años. Consideraciones finales: La IAS es una patología poco frecuente, no presenta síntomas específicos, lo que hace que el diagnóstico sea difícil. El profesional de la salud debe realizar una buena anamnesis y estar atento al histórico del paciente.

Palabras clave: Enfermedades de la Boca, Insuficiencia Suprarrenal, Hiperpigmentación, Neoplasias Encefálicas, Enfermedades de la Hipófisis.

\section{INTRODUÇÃO}

A produção deficiente de hormônios corticosteróides pela glândula suprarrenal causada pela destruição do córtex da suprarrenal ocasiona a Doença de Addison (DA) ou Insuficiência Adrenal Primária (IAP). São muitas as causas da, podendo destacar a destruição autoimune, infecções como a tuberculose e por últimos tumores, sendo esta a causa mais rara da doença (NEVILLE BW, et al., 2016). Trata-se de uma patologia rara, com uma prevalência de 140 por milhão nas populações ocidentais (FAN S, et al., 2013).

A DA pode ser classificada em Insuficiência Adrenal Primária (IAP) ou Insuficiência Adrenal Secundária (IAS), sendo a primeira uma afecção das glândulas supra-renais e a segunda é substancialmente pela hipófise ou doença hipotalâmica (PULZER A, et al., 2016).

O IAS é ocasionado pelo funcionamento inadequado da glândula hipófise, desenvolvendo-se pela diminuição da produção de Hormônio Adrenocorticotrófico $(\mathrm{ACTH})$, responsável pela manutenção dos níveis séricos do cortisol, causando alterações em que se tem destaque a doença metastática, a infecção, o enfarte e a irradiação (NEVILLE BW, et al., 2016; CARDOSO RT e PALMA IM, 2009).

As características clínicas da insuficiência adrenal só começam a se manifestar após a destruição do tecido glandular, só depois disso, iram se manifestar as características clínicas dessa doença, podendo ocasionar irritabilidade, depressão, fraqueza e fadiga. Uma hiperpigmentação ocorre na pele, mais proeminente em regiões de pontos sobre pressão e pele exposta pelo sol, ocasionada pelos níveis altos de beta-lipotropina ou ACTH (hormônio adrenocorticotrófico), ambos os hormônios têm a capacidade de excitar os melanócitos. Geralmente pode ocorrer queixas referentes a distúrbios gastrointestinais, como por exemplo anorexia, vômito, náuseas, diarreia, perda de peso e um desejo de comer sal (NEVILLE BW, et al., 2016).

Os Meningiomas são tumores benignos, de origem das células meningioendoteliais aracnoideas. Correspondem a $25 \%$ dos tumores cerebrais, a prevalência em mulheres é mais comum e são mais frequentes entre quarta e quinta década (VILAR L, et al., 2009).

O diagnóstico se dá por imagem de ressonância magnética, em alguns casos a angiografia cerebral pode auxiliar no diagnóstico, quando demonstra um blush, devido à grande vascularização do tumor (VILAR L, et al., 2009).

O tratamento padrão é cirúrgico, quanto mais completa a remoção do tumor, menores a chances de recidiva e melhores serão as chances de cura dessa patologia. Existe também o tratamento radioterápico comumente apontado como padrão em meningiomas atípicos, malignos ou recorrentes. Uma opção para tumores que estão localizados na base do crânio é a radiocirurgia, uma associação entre os dois tratamentos, que consiste em remoção parcial do tumor e posterior tratamento radioterápico (MAROSI C, et al., 2008). 
O caso descrito, tem como objetivo avaliar um caso de IAS associada a um Meningioma Psamomatoso (MP) na região da hipófise, analisando as manifestações orais da IAS, a relação entre essas patologias e discutir os dados clínicos com a literatura. A importância deste estudo é a de fornecer informações acerca da IAS associada a MP, que por se constituir num achado incomum, irá permitir aos profissionais da área da saúde um aprimoramento a respeito da associação entre essas patologias.

\section{RELATO DE CASO}

Participante da pesquisa, gênero feminino, feoderma, cinquenta e três anos de idade, compareceu ao Centro de Especialização Odontológica, queixando-se de "Apareceu umas manchas escuras em minha boca". Durante a anamnese a paciente relata que as pigmentações intrabucais apareceram a cerca de seis anos.

Durante o exame físico extrabucal, a paciente não apresentava nenhuma alteração. Já no exame intrabucal, foi observado uma lesão plana, rugosa, pigmentação melanocítica, localizada em mucosa jugal, língua e palato (Figuras 1 A, B e C). Relata ainda queixas de desconforto no estômago, devido a problemas gastrointestinais.

Figura 1 - Fotografias intrabucais mostrando os locais que foram observadas a hiperpigmentação bucal $(\rightarrow)$.

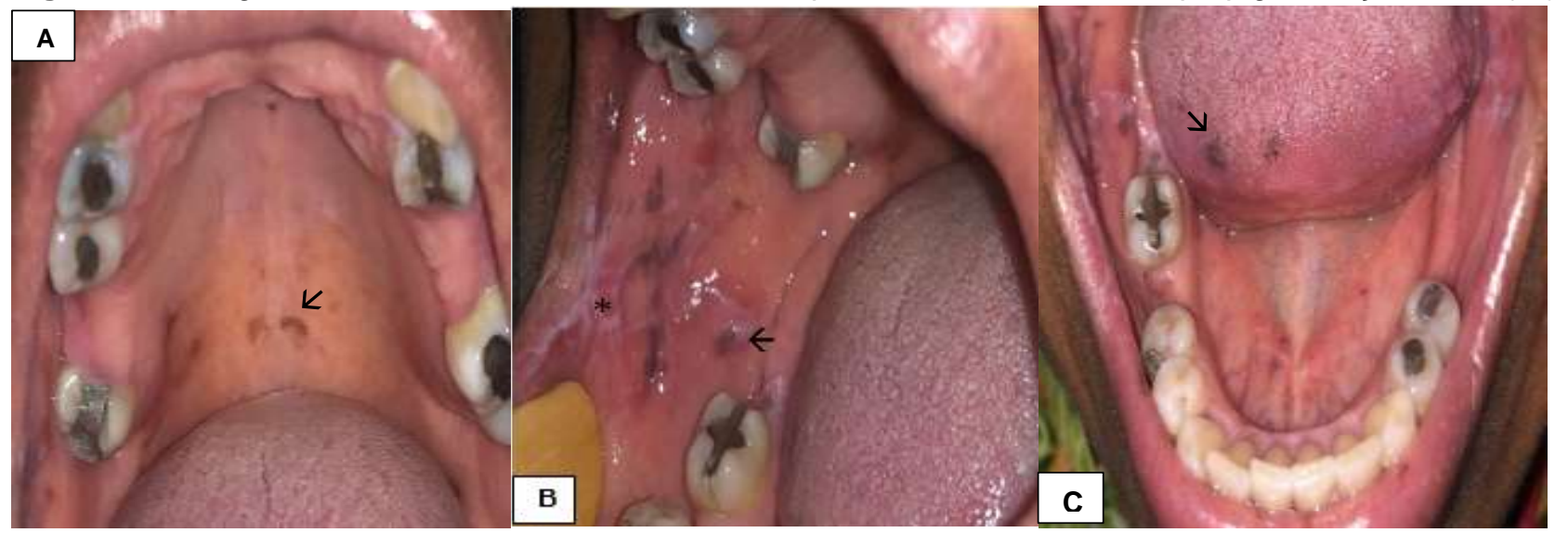

Legenda: A, trata-se de uma hiperpigmentação localizada na região do palato. B, trata-se de uma hiperpigmentação na região da mucosa jugal próxima à estriações do líquen plano oral $\left({ }^{*}\right)$. C, trata-se de uma hiperpigmentação na região do ápice da língua.

Fonte: Panjwani CM, Rocha DC, Silva KF, et al, 2018.

Quando questionada sobre a história médica, a paciente relatou ter sido diagnóstica com meningioma psamomatoso na região da hipófise há cerca de dez anos, passou por tratamento cirúrgico e radioterápico. Atualmente ela faz acompanhamento médico anualmente para controle evolutivo do tumor. Além do MP apresenta histórico de gastrite crônica há cerca de dez anos.

Após o exame clínico foi realizada uma biópsia incisional de lesão localizada em mucosa jugal do lado direito. Recebido para exame foi fixado em formalina, 1 fragmento em tecido mole medindo $0,7 \times 0,6 \mathrm{~cm}$ apresentando lesão plana e brancacenta, medindo $0,4 \times 0,3 \mathrm{~cm}$, distando $0,2 \mathrm{~cm}$. Fragmentos representativos foram submetidos a exame histológico.

Os cortes histológicos corados em HE (hematoxilina e eosina) revelam fragmentos de mucosa revestidos por epitélio estratificado pavimentoso com áreas de acantose, atrofia, hiperqueratose, espongiose, exocitose e áreas focais de degeneração hidrópica de camada basal.

Na lâmina própria subjacente era possível observar áreas de infiltrado inflamatório linfoplasmocitário rico em linfócitos dispostos em faixas destruindo focalmente a camada basal. Proeminente incontinência pigmentar localizada na área subepitelial está presente em toda a extensão dos cortes (Figura 2 A e B). 
Figura 2 - Fotomicroscopia de corte histológico da lesão biopsiada da mucosa jugal.
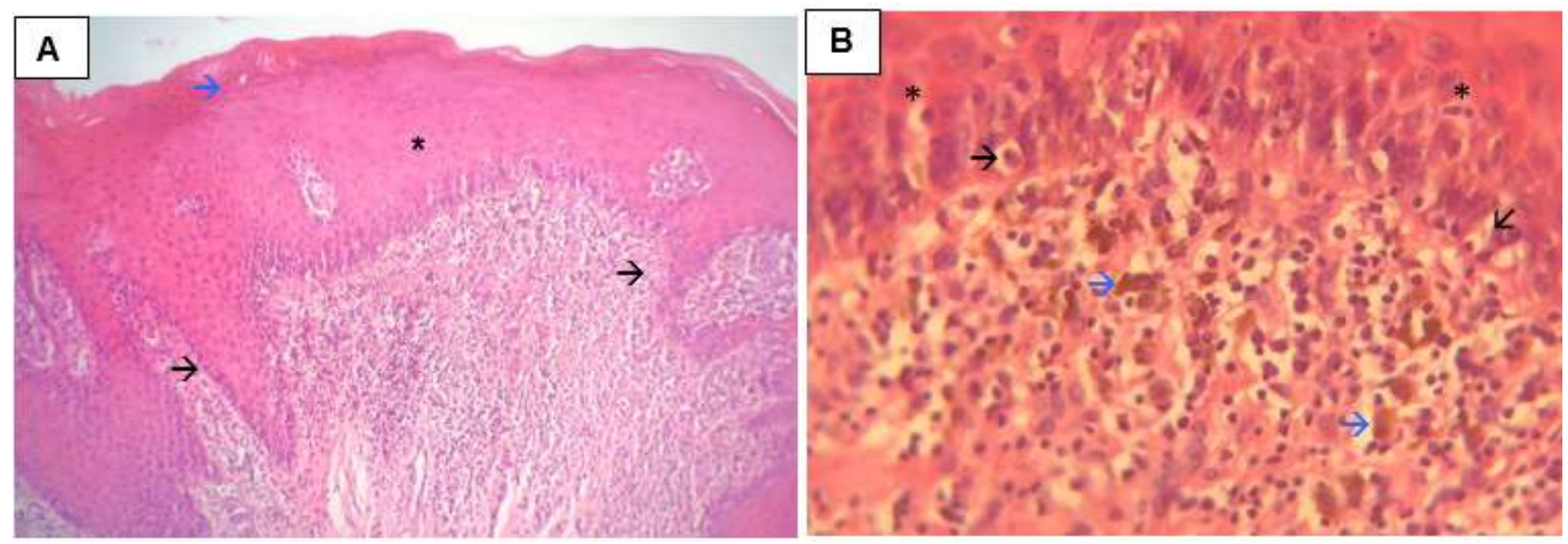

Legenda: A, Fragmento de mucosa corado em HE (hematoxilina e eosina) em um aumento de x100 mostrando hiperceratose $(\rightarrow)$, acantose $\left(^{*}\right)$, projeções em dentes de serra $(\rightarrow)$. B. Fragmento de mucosa corado em HE (hematoxilina e eosina) em um aumento de x400 evidenciando exocitose $\left(^{*}\right)$, degeneração hidrópica da camada basal $(\rightarrow)$, incotinência pigmentar $(\rightarrow)$.

Fonte: Panjwani CM, Rocha DC, Silva KF, et al, 2018.

A conclusão do exame histopatológico das lesões pigmentadas bucais, associada a dados clínicos de disfunção hormonais, trouxe as seguintes hipóteses diagnosticas: doença de Addison, ou sindrômicas (PeutzJeghers; McCuneAllbright).

Foi solicitado um exame complementar do ACTH para verificar os níveis do hormônio na paciente, onde foi encontrado o resultado de $6,9 \mathrm{pg} / \mathrm{mL}$ (progressão geométrica por massa molecular), cujo valor de referência é inferior a $46,0 \mathrm{pg} / \mathrm{mL}$, foi comprovado baixos níveis da produção do hormônio, já que o tumor estava localizado na hipófise, glândula responsável pela excreção do ACTH, característica do IAS.

Com base nos dados clínicos e histórico da participante da pesquisa de tumor hipofisário, obteve-se o diagnóstico de IAS causada pelo tumor. A participante da pesquisa foi orientada sobre a patologia e encaminhada para tratamento endocrinológico. $\mathrm{O}$ artigo descrito tem como finalidade descrever o diagnóstico uma vez que teve manifestação bucal, em razão de o tratamento está voltado para a área médica.

\section{DISCUSSÃO}

A IAS resulta de uma falência hipofisária em secretar o principal hormônio estimulador do o córtex adrenal, o ACTH. A deficiência de ACTH pode ser provocada por doença hipofisária ou hipotalâmica; neste último caso, ocorre diminuição da secreção do hormônio liberador de ACTH pelo hipotálamo. A presença de tumores na região hipotálamo-hipofisária ou o seu tratamento cirúrgico ou radioterápico são importantes causa de IA por déficit de ACTH (FREIRE DS e CATÂNIA M, 2009).

Achados na literatura ressaltam que a IAP tem como característica clínica a hiperpigmentação desencadeada pelos níveis altos de ACTH (ARLT W e ALLOLIO B, 2003; BRANT FILHO AC, et al., 2005; FELGUEIRAS HCR, 2011; FREIRE DS e CATÂNIA M, 2009; GATTI RF, et al., 2017; NEVILLE BW, et al., 2016). Todavia o caso exposto contraria estes achados, com presença de hiperpigmentação na mucosa oral e o ACTH de $6,9 \mathrm{pg} / \mathrm{mL}$ determinando um IAS.

Um estudo feito por Michels A e Michels N (2014), expôs os sinais e sintomas mais prevalentes da anorexia presente em (100\%), fadiga (100\%), hiperpigmentação (94\%) e a problemas gastrointestinais (92\%). A hiperpigmentação é o aspecto mais evidente da, como se trata de uma característica física, porém é uma característica predominantemente da primária devido aos níveis altos de ACTH nesse grupo (NEVILLE BW, et al., 2016). Achados que vão de encontro ao caso descrito, já que apesar da participante relatar problemas 
gastrointestinais, que é sinais e sintomas tanto de IAP e IAS a hiperpigmentação nesse relato não se deu pela diminuição do ACTH, como constatado pelo exame complementar solicitado para diagnóstico que evidenciou baixos níveis do hormônio.

Preto C, et al. (2018), descreve que o IAP não apresentou hiperpigmentação cutânea, embora em cerca de $94 \%$ dos casos apresente, a explicação para a ausência da hiperpigmentação no caso discutido pode ser explicado pela recente descoberta da doença no paciente relatado, já que as manifestações só começam a aparecer após 90\% destruição do tecido glandular (NEVILLE BW, et al., 2016). Como no caso apresentado esse foi um fator que dificultou o diagnóstico, já que a insuficiência adrenal é do tipo secundário e teve manifestação de hiperpigmentação, fator que pode ser desencadeado com o aumento do ACTH característica contraria ao IAS.

Em um relato de três casos clínicos de DA Brant Filho AC, et al. (2005), caracterizaram a hiperpigmentação bucal mais predominantemente encontradas na região de mucosa bucal acometendo essencialmente a língua, lábio inferior, palato duro e gengiva, as mesmas características podem ser observadas no caso relatado, entretanto não menciona relação com a IAS.

O aparecimento de lesões pigmentadas múltiplas em mucosa oral pode acontecer numa série de doenças, a hiperpigmentação associada a desordens hormonais como citado no histopatológico traz hipóteses diagnósticas de DA, além de doenças sindrômicas como a síndrome de Peutz-Jeghers e McCuneAllbright. Valendo constatar ainda, o líquen plano pigmentado e o uso contraceptivos orais, como condições que também podem desencadear essa característica.

A síndrome de Peutz-Jeghers é doença autossômica dominante e a manifestação das lesões pigmentadas acontecem no nascimento ou nos primeiros meses de vida (ALVES L, et al., 2013). No entanto foi descartada essa possibilidade, já que as lesões pigmentadas encontradas nesse relato tiveram manifestação na quinta década de vida. Enquanto isso, a síndrome McCuneAllbright é comumente determinada por uma tríade clínica, de displasia fibrosa dos ossos, manchas café-com-leite e puberdade precoce (DUMITRESCU CE e COLLINS MT, 2008). Aspectos clínicos divergentes aos encontrados já que a paciente só apresenta hiperpigmentação entre essas características.

Segundo Parada MB, et al. (2009), o líquen plano pigmentado é uma patologia que também manifesta hiperpigmentação, com ocorrência em locais de flexura, pescoço e face, não sendo vista em mucosa bucal (CONTIN LA, et al., 2014). Descartando então essa possibilidade para diagnostico do caso descrito, valendo constatar assim as pigmentações só em região de cavidade oral.

O uso sistêmico e por longos períodos de contraceptivos orais como tranquilizantes, anticoncepcionais orais, citostáticos, hidantoínas e antimaláricos podem induz à formação de regiões pigmentadas em mucosa oral, que somem após a interrupção dos medicamentos (RODRIGO G, et al., 2004; WECKX LLM, et al., 1995). Embora exista relato do uso de um desses contraceptivos por um curto período de tempo, as manifestações orais já existiam e permanecem até então, mesmo cessando o uso do medicamento.

A IAS tem como principal decorrência tumores na região hipotálamo-hipófise, frequentemente está associada a desordens causadas a pituitária, devido ao crescimento do tumor ou tratamento do mesmo com cirurgia ou irradiação (ARLT W e ALLOLIO B, 2003). Bem como os antecedentes encontrados na participante da pesquisa, que relata histórico de cirurgia para ressecção do MP e radioterapia.

A associação do meningioma se deve ao fato que a IAS pode ser causada por doenças que comprometem o eixo hipotalamoipofisário como é o caso do meningioma psamomatoso (CASTRO M e ELIAS LL, 2003). No caso relatado o MP estava localizado na região da hipófise, fator que contribuiu para achados de baixos níveis de ACTH na participante da pesquisa, visto que a hipófise é responsável pela secreção desse hormônio e estava comprometida pelo tumor.

Uma revisão retrospectiva realizada por Sathananthan M, et al. (2013), avaliou entre 1975 e 2003 o prontuário de 17 pacientes diagnosticados com meningiomas puramente ou intrasselar tratados cirurgicamente na Mayo Clinic. A cura cirúrgica foi alcançada em $53 \%$ desses pacientes, porém evidenciou- 
se que o hipopituarismo pós-cirúrgico, que é a diminuição da secreção hormonal da glândula pituitária acometeu em alta frequência esses pacientes. O caso exposto também se correlaciona com esses achados, já que a participante da pesquisa passou por procedimentos cirúrgicos e radioterápicos com a finalidade de alcançar a cura para o MP e posteriormente apresentou IAS.

O IAS é uma patologia pouco frequente, não apresenta sintomas específicos, o que torna o diagnóstico difícil. O profissional da saúde deve realizar uma boa anamnese e ficar atento ao histórico do paciente como por exemplo o tratamento prolongado com corticoide, infecções ou traumatismos do sistema nervoso central ou tumores na região da hipófise por exemplo o MP, achados que podem auxiliar no diagnóstico. Além disso, a manifestação da hiperpigmentação em mucosa bucal se caracteriza como um achado incomum em pacientes com IAS.

\section{REFERÊNCIAS}

1. ALVES L, et al. Peutz Jeghers: relato de caso. Revista Científica da Faculdade de Medicina de Campos, 2013; 8(2): 16-21.

2. ARLT W, ALLOLIO B. Adrenal insufficiency. The Lancet, 2003; 361(9372): 1881-1893.

3. BRANT FILHO AC, et al. Doença de Addison: diagnóstico, patogenia e implicações odontológicas. Relato de três casos clínicos. Revista de Pós-Graduação, 2005; 12(2): 270-275.

4. CARDOSO RT e PALMA IM. Insuficiência do cortex supra-renal: fisiopatologia, diagnóstico e tratamento. Revista Portuguesa de Endocrinologia, Diabetes e Metabolismo, 2009; 1: 77-87.

5. CASTRO M e ELIAS LL. Insuficiência adrenal crônica e aguda. Medicina (Ribeirão Preto. Online), 2003; 36(2/4): 375-379.

6. CONTIN LA, et al. Líquen plano pigmentoso: tratamento cirúrgico com dermoabrasão. Surgical \& Cosmetic Dermatology, 2014; 6(4): 384-386.

7. DUMITRESCU CE e COLLINS MT. Mccune-albright syndrome. Orphanet journal of rare diseases, 2008; 3(1): 12.

8. FAN S, et al. Pituitary ACTH-secreting adenoma in Addison's disease: a case report. Clinical neurology and neurosurgery, 2013; 115(12): 2543.

9. FELGUEIRAS HCR. Insuficiência Adrenal Secundária-dificuldades de diagnóstico. Dissertação (Mestrado em Medicina) - Instituto de Ciências Abel Salazar. Universidade do Porto, Porto, 2011; 5 p.

10. INSUFICIÊNCIA ADRENAL. 2009. In: MEDICINA net. Disponível em: http://assinantes.medicinanet.com.br/conteudos/revisoes/1300/insuficiencia adrenal.htm. Acesso em: 10 mar. 2019.

11. GATTI RF, et al. Hiperpigmentação na Doença de Addison - Relato De Caso. Journal of the Portuguese Society of Dermatology and Venereology, 2017; 75(2): 169-172.

12. MAROSI C, et al. Meningioma. Critical reviews in oncology/hematology, 2008; 67(2): 153-171.

13. MICHELS A e MICHELS N. Addison disease early detection and treatment principles. Am Fam Physician, 2014; 89(07): 509.

14. NEVILLE BW, et al. Patologia oral e maxilofacial. 4nd ed. Rio de Janeiro: Elsevier, 2016; 844-845 p.

15. PARADA MB, et al. Tratamento do líquen plano pigmentoso com luz intensa pulsada. Surgical \& Cosmetic Dermatology, v. 1, n. 4, p. 193-195, 2009.

16. PRETO C, et al. Doença de Addison: a dificuldade de diagnóstico. Nascer e Crescer, 2018; 27(1): 39-42.

17. PULZER A, et al. Addison's disease: Primary adrenal insufficiency. Der Internist, 2016; 57(5): 457-469.

18. RODRIGO RG, et al. As principais lesões enegrecidas da cavidade oral. Revista Cubana de Estomatologia, 2014; 51(2): 195-201.

19. SATHANANTHAN M, et al. Sellar meningiomas: an endocrinologic perspective. Pituitary, 2013; 16(2), 182-188.

20. VILAR L, et al. Endocrinologia clínica. 4nd ed. Rio de Janeiro: Guanabara Koogan, 2009; 7 p.

21. WECKX L, et al. Pigmentação bucal e anemia. Apresentação de um caso. Revista Brasileira de Otorrinolaringologia, 1995; 61(3), 246-248. 